\title{
Is social media really impacting urogynecology?
}

\section{Luiz Gustavo Oliveira Brito ${ }^{1}$}

Published online: 30 May 2020

(C) The International Urogynecological Association 2020

Social media (SoMe) is increasingly being embraced by researchers in medicine. Medical journals have begun to understand the power of SoMe to interact with their readers online and to promote their articles [1]. Moreover, the medical community has been actively using SoMe to get connected and to exchange ideas. Academics can share their research with a broader audience [2]. In urogynecology, there has been a rapid increase in the usefulness of social media by health professionals [3]. Finally, patients are getting more connected with $\mathrm{SoMe}$, especially women presenting to urogynecology practices; they have high internet use and a desire to learn about their diseases [4].

Among several SoMe platforms, Twitter is the preferred one because of its succinct input and rapid conversations (limited to 280 characters). If you decide to summarize any article or topic, you can create chains of connected tweets, called a "thread." When a thread from a medical journal is discussing a specific topic, such as an article, this thread can be denominated as a "tweetorial," and this gets a lot of attention from the readers because it is a summary of a study that can be shared (or "retweeted") with everyone. These topics can be easily identified by hashtags (e.g., \#urogynecology), and this process facilitates the access of information to anyone who is interested in this topic.

Instagram is another SoMe platform, mainly visual, and can be used for interviews or to display visual abstracts; it can also stream "live transmission" for two people. Facebook also allows long messages for each post as well as video and live stream transmissions. Podcasts (usually audio conversations) are another internet-based medical resource, and their use and application in urogynecology were extensively discussed in a previous IUJ article by Chen and Melon [5].

Luiz Gustavo Oliveira Brito

lgobrito@gmail.com

1 Department of Obstetrics and Gynecology, School of Medical Sciences, University of Campinas, Rua Alexander Fleming, 101 Cidade Universitária, Campinas 13083-881, Brazil
Whether social media has an impact on article citations is under discussion, and many studies have been trying to establish a causal relation. One index that was created to measure social attention toward scientific research was the alternativelevel metrics (Altmetrics), a weighted composite score that includes SoMe platforms, Wikipedia, Mendeley, policy documents, and traditional media sharing (blogs, news) [6]. Another format to analyze SoMe's impact is when we compare a researcher's impact on SoMe to their number of citations. The Kardashian index is one of these measures and consists of plotting the number of Twitter followers against the number of citations an individual has [7]. A study published by Chandrasekar et al. [8] with academic urologists and programs in North America has shown that physicians with higher $\mathrm{H}$-indices and citations were associated with a higher number of followers; however, they were less likely to engage other followers with "likes" (one-way engagement).

When we analyze whether or not SoMe increases citations, it seems it might be true as well. In coloproctology, article exposure on Twitter was strongly associated with a high citation level $(\mathrm{OR}=8.6, p=0.001)$ [9]. Hayon et al. [10] analyzed 230 papers from seven urologic journals, and they found that articles mentioned on Twitter had 2-fold more Scopus citations and 2.3-fold more Google Scholar citations compared with articles with no Twitter mentions. Moreover, female urologic articles had the most Twitter mentions. Another study from Calopedos et al. [11] found a correlation between the number of citations and Altmetric scores $(r=0.72$, $p<0.001$ ); this study also concluded that urologic articles were often cited, particularly in the non-urologic literature. Another study on urogynecology terms in SoMe found that the Altmetric Attention Score (AAS) is especially influenced by Twitter [12]. Sathianathen et al. [13] developed a urology social media score (\#UroSoMe_Score) to predict citation counts from measures of online attention for urologic articles. They found an association between the Altmetric score and 2year Scopus citation counts $(p<0.001)$. Moreover, citations could be predicted from a model comprising policy documents, Google+, blogs, videos, Wikipedia, Twitter, and Q\&A. But this still needs further data. 
It is important to understand that SoMe may have its collateral effects: a systematic review found 57 articles discussing health-related misinformation and the role of SoMe in its propagation, mostly related to infectious diseases [14]; this underscores the importance of spreading solid and concrete information, especially in these times of the COVID19 pandemic, and especially for fellow SoMe editors at other medical journals [15].

The Blue Journal started a SoMe account 2 years ago, and we currently have 700+ followers on Twitter (twitter.com/ IUJ_BlueJournal) as well as $1776+$ Facebook followers (facebook.com/IUJBlueJournal) and 245+ Instagram followers (www.instagram.com/iuj_bluejournal). We are happy to deliver information to our readers and from our SoMe platforms; all shared articles within our posts present a shareable link that makes all content readable, thanks to our publisher's initiative. We invite you to like and follow our Facebook, Twitter, and Instagram accounts and to contribute to our journal SoMe by discussing our manuscripts, sharing our visual abstracts/studies, and adding your voice to our SoMe accounts.

Acknowledgments I thank Rebecca Rogers and Steve Swift's initiative for launching an IUJ SoMe account and the help of other peers, such as Bobby Garcia and, last but not least, the indispensable aid of Lindsay MacMurray, IUJ's Managing Editor.

\section{Compliance with ethical standards}

Conflict of interests The author reports no conflict of interests.

\section{References}

1. Snipelisky D. Social media in medicine: a podium without boundaries. J Am Coll Cardiol. 2015;65(22):2459-61.

2. Klar S, Krupnikov Y, Ryan JB, Searles K, Shmargad Y. Using social media to promote academic research: identifying the benefits of twitter for sharing academic work. PLoS One. 2020;15(4): $\mathrm{e} 0229446$.

3. Alas A, Sajadi KP, Goldman HB, Anger JT. The rapidly increasing usefulness of social media in urogynecology. Female Pelvic Med Reconst Surg. 2013;19(4):210-3.

4. Mazloomdoost D, Kanter G, Chan RC, Deveaneau N, Wyman AM, Von Bargen AC, et al. Social networking and internet use among pelvic floor patients: a multicentrer survey. Am J Obstet Gynecol. 2016;654:e1-e10. https://doi.org/10.1016/j.ajog.2016.06.011.

5. Chen Z, Melon J. Evolution of social media: review of the role of podcasts in gynecology. Int Urogynecol J. 2018;29:477-80.

6. Trueger NS, Thoma B, Hsu CH, Sullivan D, Peters L, Lin M. The altmetric score: a new measure for article-level dissemination and impact. Ann Emerg Med. 2015;66:549-53.

7. Hall N. The Kardashian index: a measure of discrepant social media profile for scientists. Genome Biol. 2014;15:424.

8. Chandrasekar T, Goldberg H, Klaassen Z, Wallis CJ, Leong JY, Liem S, et al. Twitter and academic urology in the United States and Canada: a comprehensive assessment of the Twitterverse in 2019. BJU Int. 2020;125:173-81.

9. Jeong JW, Kim MJ, Oh HK, et al. The impact of social media on citation rates in coloproctology. Color Dis. 2019;21:1175-82.

10. Hayon S, Tripathi H, Stormont IM, Dunne MM, Naslund MJ, Siddiqui MM. Twitter mentions and academic citations in the urologic literature. Urology. 2019;123:28-33.

11. Calopedos RJ, Garcia C, Rashid P, Murphy DG, Lawrentschuk N, Woo HH. Citation indices for social media articles in urology. BJU Int. 2017;119:S5,47-52.

12. Varella G, Juliato CR. Brito LG (2019) assessing the impact of urogynecology research in social media: are we being read ? Female Pelvic Med Reconstr Surg. 2019;25:S193.

13. Sathianathen NJ, Lane R 3rd, Condon B. Early online attention can predict citation counts for urological publicatons: the \#urosome_score. Eur Urol Focus. 2019. https://doi.org/10.1016/j. euf.2019.10.015.

14. Wang Y, McKee M, Torbica A, Stuckler D. Systematic literature review on the spread of health-related misinformation on social media. Soc Sci Med. 2019;240:112552.

15. Lopes M, Chan TM, Thoma B, Arora VM, Trueger NS. The social media editor at medical journals: responsibilities, goals, barriers, and facilitators. Acad Med. 2019;94(5):701-7.

Publisher's note Springer Nature remains neutral with regard to jurisdictional claims in published maps and institutional affiliations. 